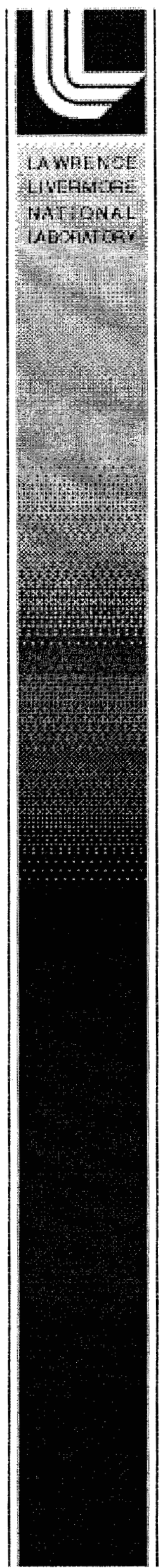

\title{
Rates, Polarizations, and Asymmetries in Charmless Vector-Vector B Decays
}

The BARBAR Collaboration, et al., D Wright

March 11, 2003

This article was submitted to $X V I^{\text {th }}$ Rencontres de la Vallee d'Aoste, March 9-15, 2003, La Thuile, Vallee d'Aoste, Italy 


\section{DISCLAIMER}

This document was prepared as an account of work sponsored by an agency of the United States Government. Neither the United States Government nor the University of California nor any of their employees, makes any warranty, express or implied, or assumes any legal liability or responsibility for the accuracy, completeness, or usefulness of any information, apparatus, product, or process disclosed, or represents that its use would not infringe privately owned rights. Reference herein to any specific commercial product, process, or service by trade name, trademark, manufacturer, or otherwise, does not necessarily constitute or imply its endorsement, recommendation, or favoring by the United States Government or the University of California. The views and opinions of authors expressed herein do not necessarily state or reflect those of the United States Government or the University of California, and shall not be used for advertising or product endorsement purposes.

This is a preprint of a paper intended for publication in a journal or proceedings. Since changes may be made before publication, this preprint is made available with the understanding that it will not be cited or reproduced without the permission of the author.

This work was performed under the auspices of the United States Department of Energy by the University of California, Lawrence Livermore National Laboratory under contract No. W-7405-Eng-48.

This report has been reproduced directly from the best available copy.

Available electronically at http://www.doc.gov/bridge

Available for a processing fee to U.S. Department of Energy

And its contractors in paper from

U.S. Department of Energy

Office of Scientific and Technical Information

P.O. Box 62

Oak Ridge, TN 37831-0062

Telephone: (865) 576-8401

Facsimile: (865) 576-5728

E-mail: reports@adonis.osti.gov

Available for the sale to the public from

U.S. Department of Commerce

National Technical Information Service

5285 Port Royal Road

Springfield, VA 22161

Telephone: (800) 553-6847

Facsimile: (703) 605-6900

E-mail: orders@ntis.fedworld.gov

Online ordering: http://www.ntis.gov/ordering.htm

OR

Lawrence Livermore National Laboratory

Technical Information Department's Digital Library

http://www.llnl.gov/tid/Library.html 
The BABAR Collaboration,

B. Aubert, R. Barate, D. Boutigny, J.-M. Gaillard, A. Hicheur, Y. Karyotakis, J. P. Lees, P. Robbe, V. Tisserand, A. Zghiche

Laboratoire de Physique des Particules, F-74941 Annecy-le-Vieux, France

A. Palano, A. Pompili

Università di Bari, Dipartimento di Fisica and INFN, I-70126 Bari, Italy

J. C. Chen, N. D. Qi, G. Rong, P. Wang, Y. S. Zhu

Institute of High Energy Physics, Beijing 100039, China

G. Eigen, I. Ofte, B. Stugu

University of Bergen, Inst. of Physics, N-5007 Bergen, Norway

G. S. Abrams, A. W. Borgland, A. B. Breon, D. N. Brown, J. Button-Shafer, R. N. Cahn, E. Charles, C. T. Day, M. S. Gill, A. V. Gritsan, Y. Groysman, R. G. Jacobsen, R. W. Kadel, J. Kadyk, L. T. Kerth, Yu. G. Kolomensky, J. F. Kral, G. Kukartsev, C. LeClerc, M. E. Levi, G. Lynch, L. M. Mir, P. J. Oddone,

T. J. Orimoto, M. Pripstein, N. A. Roe, A. Romosan, M. T. Ronan, V. G. Shelkov, A. V. Telnov, W. A. Wenzel

Lawrence Berkeley National Laboratory and University of California, Berkeley, CA 94720, USA

T. J. Harrison, C. M. Hawkes, D. J. Knowles, R. C. Penny, A. T. Watson, N. K. Watson University of Birmingham, Birmingham, B15 2TT, United Kingdom

T. Deppermann, K. Goetzen, H. Koch, B. Lewandowski, M. Pelizaeus, K. Peters, H. Schmuecker, M. Steinke

Ruhr Universität Bochum, Institut für Experimentalphysik 1, D-44780 Bochum, Germany

N. R. Barlow, W. Bhimji, J. T. Boyd, N. Chevalier, W. N. Cottingham, C. Mackay, F. F. Wilson

University of Bristol, Bristol BS8 1TL, United Kingdom

C. Hearty, T. S. Mattison, J. A. McKenna, D. Thiessen

University of British Columbia, Vancouver, BC, Canada V6T $1 Z 1$

P. Kyberd, A. K. McKemey

Brunel University, Uxbridge, Middlesex UB8 3 PH, United Kingdom

V. E. Blinov, A. D. Bukin, V. B. Golubev, V. N. Ivanchenko, E. A. Kravchenko, A. P. Onuchin, S. I. Serednyakov, Yu. I. Skovpen, E. P. Solodov, A. N. Yushkov

Budker Institute of Nuclear Physics, Novosibirsk 630090, Russia

D. Best, M. Chao, D. Kirkby, A. J. Lankford, M. Mandelkern, S. McMahon, R. K. Mommsen, W. Roethel, D. P. Stoker

University of California at Irvine, Irvine, CA 92697, USA

C. Buchanan

University of California at Los Angeles, Los Angeles, CA 90024, USA

H. K. Hadavand, E. J. Hill, D. B. MacFarlane, H. P. Paar, Sh. Rahatlou, U. Schwanke, V. Sharma. University of California at San Diego, La Jolla, CA 92093, USA 
J. W. Berryhill, C. Campagnari, B. Dahmes, N. Kuznetsova, S. L. Levy, O. Long, A. Lu, M. A. Mazur, J. D. Richman, W. Verkerke

University of California at Santa Barbara, Santa Barbara, CA 93106, USA

J. Beringer, A. M. Eisner, C. A. Heusch, W. S. Lockman, T. Schalk, R. E. Schmitz, B. A. Schumm,

A. Seiden, M. Turri, W. Walkowiak, D. C. Williams, M. G. Wilson

University of California at Santa Cruz, Institute for Particle Physics, Santa Cruz, CA 95064, USA

J. Albert, E. Chen, M. P. Dorsten, G. P. Dubois-Felsmann, A. Dvoretskii, D. G. Hitlin, I. Narsky,

F. C. Porter, A. Ryd, A. Samuel, S. Yang

California Institute of Technology, Pasadena, CA 91125, USA

S. Jayatilleke, G. Mancinelli, B. T. Meadows, M. D. Sokoloff

University of Cincinnati, Cincinnati, $O H$ 45221, USA

T. Barillari, F. Blanc, P. Bloom, P. J. Clark, W. T. Ford, U. Nauenberg, A. Olivas, P. Rankin, J. Roy, J. G. Smith, W. C. van Hoek, L. Zhang

University of Colorado, Boulder, CO 80309, USA

J. L. Harton, T. Hu, A. Soffer, W. H. Toki, R. J. Wilson, J. Zhang

Colorado State University, Fort Collins, CO 80523, USA

D. Altenburg, T. Brandt, J. Brose, T. Colberg, M. Dickopp, R. S. Dubitzky, A. Hauke, H. M. Lacker, E. Maly, R. Müller-Pfefferkorn, R. Nogowski, S. Otto, K. R. Schubert, R. Schwierz, B. Spaan, L. Wilden Technische Universität Dresden, Institut für Kern- und Teilchenphysik, D-01062 Dresden, Germany

D. Bernard, G. R. Bonneaud, F. Brochard, J. Cohen-Tanugi, Ch. Thiebaux, G. Vasileiadis, M. Verderi Ecole Polytechnique, LLR, F-91128 Palaiseau, France

A. Khan, D. Lavin, F. Muheim, S. Playfer, J. E. Swain, J. Tinslay

University of Edinburgh, Edinburgh EH9 $3 J Z$, United Kingdom

C. Bozzi, L. Piemontese, A. Sarti

Università di Ferrara, Dipartimento di Fisica and INFN, 1-44100 Ferrara, Italy

E. Treadwell

Florida A6M University, Tallahassee, FL 32307, USA

F. Anulli, ${ }^{1}$ R. Baldini-Ferroli, A. Calcaterra, R. de Sangro, D. Falciai, G. Finocchiaro, P. Patteri, I. M. Peruzzi, ${ }^{1}$ M. Piccolo, A. Zallo

Laboratori Nazionali di Frascati dell'INFN, I-00044 Frascati, Italy

A. Buzzo, R. Contri, G. Crosetti, M. Lo Vetere, M. Macri, M. R. Monge, S. Passaggio, F. C. Pastore, C. Patrignani, E. Robutti, A. Santroni, S. Tosi

Università di Genova, Dipartimento di Fisica and INFN, I-16146 Genova, Italy

S. Bailey, M. Morii

Harvard University, Cambridge, MA 02138, USA

\footnotetext{
${ }^{1}$ Also with Università di Perugia, Perugia, Italy
} 

G. J. Grenier, S.-J. Lee, U. Mallik
University of Iowa, Iowa City, IA 52242, USA
J. Cochran, H. B. Crawley, J. Lamsa, W. T. Meyer, S. Prell, E. I. Rosenberg, J. Yi
Iowa State University, Ames, IA 50011-3160, USA

M. Davier, G. Grosdidier, A. Höcker, S. Laplace, F. Le Diberder, V. Lepeltier, A. M. Lutz, T. C. Petersen, S. Plaszczynski, M. H. Schune, L. Tantot, G. Wormser Laboratoire de l'Accélérateur Linéaire, F-91898 Orsay, France

R. M. Bionta, V. Brigljević, C. H. Cheng, D. J. Lange, D. M. Wright

Lawrence Livermore National Laboratory, Livermore, CA 94550, USA

A. J. Bevan, J. R. Fry, E. Gabathuler, R. Gamet, M. Kay, D. J. Payne, R. J. Sloane, C. Touramanis University of Liverpool, Liverpool L69 $3 B X$, United Kingdom

M. L. Aspinwall, D. A. Bowerman, P. D. Dauncey, U. Egede, I. Eschrich, G. W. Morton, J. A. Nash, P. Sanders, G. P. Taylor

University of London, Imperial College, London, $S W 72 B W$, United Kingdom

J. J. Back, G. Bellodi, P. F. Harrison, H. W. Shorthouse, P. Strother, P. B. Vidal Queen Mary, University of London, E1 4NS, United Kingdom

G. Cowan, H. U. Flaecher, S. George, M. G. Green, A. Kurup, C. E. Marker, T. R. McMahon, S. Ricciardi, F. Salvatore, G. Vaitsas, M. A. Winter

University of London, Royal Holloway and Bedford New College, Egham, Surrey TW20 OEX, United Kingdom

D. Brown, C. L. Davis

University of Louisville, Louisville, KY 40292, USA

J. Allison, R. J. Barlow, A. C. Forti, P. A. Hart, F. Jackson, G. D. Lafferty, A. J. Lyon, J. H. Weatherall, J. C. Williams

University of Manchester, Manchester M13 9PL, United Kingdom

A. Farbin, A. Jawahery, D. Kovalskyi, C. K. Lae, V. Lillard, D. A. Roberts

University of Maryland, College Park, MD 20\%42, USA

G. Blaylock, C. Dallapiccola, K. T. Flood, S. S. Hertzbach, R. Kofler, V. B. Koptchev, T. B. Moore,

H. Staengle, S. Willocq

University of Massachusetts, Amherst, MA 01003, USA

R. Cowan, G. Sciolla, F. Taylor, R. K. Yamamoto

Massachusetts Institute of Technology, Laboratory for Nuclear Science, Cambridge, MA 02139, USA

D. J. J. Mangeol, M. Milek, P. M. Patel

McGill University, Montréal, QC, Canada H3A 2T8

A. Lazzaro, F. Palombo

Università di Milano, Dipartimento di Fisica and INFN, I-20133 Milano, Italy 
J. M. Bauer, L. Cremaldi, V. Eschenburg, R. Godang, R. Kroeger, J. Reidy, D. A. Sanders, D. J. Summers, H. W. Zhao

University of Mississippi, University, MS 38677, USA

C. Hast, P. Taras

Université de Montréal, Laboratoire René J. A. Lévesque, Montréal, QC, Canada H3C $3 J 7$

H. Nicholson

Mount Holyoke College, South Hadley, MA 01075, USA

C. Cartaro, N. Cavallo, G. De Nardo, F. Fabozzi, ${ }^{2}$ C. Gatto, L. Lista, P. Paolucci, D. Piccolo, C. Sciacca Università di Napali Federico II, Dipartimento di Scienze Fisiche and INFN, I-80126, Napoli, Italy

\section{A. Baak, G. Raven}

NIKHEF, National Institute for Nuclear Physics and High Energy Physics, 1009 DB Amsterdam, The Netherlands

J. M. LoSecco

University of Notre Dame, Notre Dame, IN 46556, USA

T. A. Gabriel

Oak Ridge National Laboratory, Oak Ridge, TN 37831, USA

B. Brau, T. Pulliam

Ohio State University, Columbus, OH 43210, USA

J. Brau, R. Frey, M. Iwasaki, C. T. Potter, N. B. Sinev, D. Strom, E. Torrence

University of Oregon, Eugene, OR 97403, USA

F. Colecchia, A. Dorigo, F. Galeazzi, M. Margoni, M. Morandin, M. Posocco, M. Rotondo, F. Simonetto, R. Stroili, G. Tiozzo, C. Voci

Università di Padova, Dipartimento di Fisica and INFN, I-35131 Padova, Italy

M. Benayoun, H. Briand, J. Chauveau, P. David, Ch. de la Vaissière, L. Del Buono, O. Hamon, Ph. Leruste, J. Ocariz, M. Pivk, L. Roos, J. Stark, S. T'Jampens

Universités Paris VI et VII, Lab de Physique Nucléaire H. E., F-75252 Paris, France

P. F. Manfredi, V. Re

Università di Pavia, Dipartimento di Elettronica and INFN, I-27100 Pavia, Italy

L. Gladney, Q. H. Guo, J. Panetta

University of Pennsylvania, Philadelphia, PA 19104, USA

C. Angelini, G. Batignani, S. Bettarini, M. Bondioli, F. Bucci, G. Calderini, M. Carpinelli, F. Forti, M. A. Giorgi, A. Lusiani, G. Marchiori, F. Martinez-Vidal, ${ }^{3}$ M. Morganti, N. Neri, E. Paoloni, M. Rama, G. Rizzo, F. Sandrelli, J. Walsh

Università di Pisa, Dipartimento di Fisica, Scuola Normale Superiore and INFN, I-56127 Pisa, Italy

${ }^{2}$ Also with Università della Basilicata, Potenza, Italy

${ }^{3}$ Also with IFIC, Instituto de Física Corpuscular, CSIC-Universidad de Valencia, Valenc ia, Spain 
M. Haire, D. Judd, K. Paick, D. E. Wagoner

Prairie View ABM University, Prairie View, TX 77/46, USA

N. Danielson, P. Elmer, C. Lu, V. Miftakov, J. Olsen, A. J. S. Smith, E. W. Varnes

Princeton University, Princeton, NJ 08544, USA

F. Bellini, G. Cavoto, ${ }^{4}$ D. del Re, R. Faccini, ${ }^{5}$ F. Ferrarotto, F. Ferroni, M. Gaspero, E. Leonardi, M. A. Mazzoni, S. Morganti, M. Pierini, G. Piredda, F. Safai Tehrani, M. Serra, C. Voena

Università di Roma La Sapienza, Dipartimento di Fisica and INFN, I-00185 Roma, Italy

S. Christ, G. Wagner, R. Waldi

Universität Rostock, D-18051 Rostock, Germany

T. Adye, N. De Groot, B. Franek, N. I. Geddes, G. P. Gopal, E. O. Olaiya, S. M. Xella

Rutherford Appleton Laboratory, Chilton, Didcot, Oxon, OX11 0QX, United Kingdom

R. Aleksan, S. Emery, A. Gaidot, S. F. Ganzhur, P.-F. Giraud, G. Hamel de Monchenault, W. Kozanecki, M. Langer, G. W. London, B. Mayer, G. Schott, G. Vasseur, Ch. Yeche, M. Zito

DAPNIA, Commissariat à l'Energie Atomique/Saclay, F-91191 Gif-sur-Yvette, France

M. V. Purohit, A. W. Weidemann, F. X. Yumiceva.

University of South Carolina, Columbia, SC 29208, USA

D. Aston, R. Bartoldus, N. Berger, A. M. Boyarski, O. L. Buchmueller, M. R. Convery, D. P. Coupal, D. Dong, J. Dorfan, D. Dujmic, W. Dunwoodie, R. C. Field, T. Glanzman, S. J. Gowdy, E. Grauges-Pous;

T. Hadig, V. Halyo, T. Hryn'ova, W. R. Innes, C. P. Jessop, M. H. Kelsey, P. Kim, M. L. Kocian,

U. Langenegger, D. W. G. S. Leith, S. Luitz, V. Luth, H. L. Lynch, H. Marsiske, S. Menke, R. Messner,

D. R. Muller, C. P. O'Grady, V. E. Ozcan, A. Perazzo, M. Perl, S. Petrak, B. N. Ratcliff, S. H. Robertson,

A. Roodman, A. A. Salnikov, R. H. Schindler, J. Schwiening, G. Simi, A. Snyder, A. Soha, J. Stelzer,

D. Su, M. K. Sullivan, H. A. Tanaka, J. Va'vra, S. R. Wagner, M. Weaver, A. J. R. Weinstein,

W. J. Wisniewski, D. H. Wright, C. C. Young

Stanford Linear Accelerator Center, Stanford, CA 94309, USA

P. R. Burchat, T. I. Meyer, C. Roat

Stanford University, Stanford, CA 94305-4060, USA

S. Ahmed, J. A. Ernst

State Univ. of New York, Albany, NY 12222, USA

W. Bugg, M. Krishnamurthy, S. M. Spanier

University of Tennessee, Knoxville, TN 37996, USA

R. Eckmann, H. Kim, J. L. Ritchie, R. F. Schwitters

University of Texas at Austin, Austin, TX 78712, USA

J. M. Izen, I. Kitayama, X. C. Lou, S. Ye

University of Texas at Dallas, Richardson, TX 75083, USA

\footnotetext{
${ }^{4}$ Also with Princeton University, Princeton, NJ 08544, USA

${ }^{5}$ Also with University of California at San Diego, La Jolla, CA 92093, USA
} 


\section{F. Bianchi, M. Bona, F. Gallo, D. Gamba}

Università di Torino, Dipartimento di Fisica Sperimentale and INFN, I-10125 Torino, Italy

C. Borean, L. Bosisio, G. Della Ricca, S. Dittongo, S. Grancagnolo, L. Lanceri, P. Poropat, ${ }^{6}$ L. Vitale, G. Vuagnin

Università di Trieste, Dipartimento di Fisica and INFN, I-34127 Trieste, Italy

R. S. Panvini

Vanderbilt University, Nashville, TN 37235, USA

Sw. Banerjee, C. M. Brown, D. Fortin, P. D. Jackson, R. Kowalewski, J. M. Roney

University of Victoria, Victoria, BC, Canada V8W $3 P 6$

H. R. Band, S. Dasu, M. Datta, A. M. Eichenbaum, H. Hu, J. R. Johnson, R. Liu, F. Di Lodovico, A. K. Mohapatra, Y. Pan, R. Prepost, S. J. Sekula, J. H. von Wimmersperg-Toeller, J. Wu, S. L. Wu, Z. Yu

University of Wisconsin, Madison, WI 53706, USA

H. Neal

Yale University, New Haven, CT 06511, USA

\footnotetext{
${ }^{6}$ Deceased
} 
BABAR Analysis Document \#576, Version 8

BABAR-CONF-03/002

SLAC-PUB- $\mathrm{xxxx}$

hep-ex/xxxx

March 2003

\title{
Rates, Polarizations, and Asymmetries in Charmless Vector-Vector $B$ Decays
}

\author{
The BABAR Collaboration
}

March 11, 2003

\begin{abstract}
With a sample of approximately 89 million $B \bar{B}$ pairs collected with the $B A B A R$ detector, we measure branching fractions, determine the degree of longitudinal polarization, and search for direct $C P$ violation in the decays $B^{0} \rightarrow \phi K^{* 0}$ and $B^{+} \rightarrow \phi K^{*+}$. We perform a search for other charmless vector-vector $B$ decays involving $\rho$ and $K^{*}(892)$ resonances and observe the decays $B^{+} \rightarrow \rho^{0} K^{*+}$ and $B^{+} \rightarrow \rho^{0} \rho^{+}$. The branching fractions are measured to be $\mathcal{B}\left(\phi K^{* 0}\right)=\left(11.1_{-1.2}^{+1.3} \pm 1.1\right) \times$ $10^{-6}, \mathcal{B}\left(\phi K^{*+}\right)=\left(12.1_{-1.9}^{+2.1} \pm 1.5\right) \times 10^{-6}, \mathcal{B}\left(\rho^{0} K^{*+}\right)=\left(7.7_{-2.0}^{+2.1} \pm 1.4\right) \times 10^{-6}$, and $\mathcal{B}\left(\rho^{0} \rho^{+}\right)=$ $\left(9.9_{-2.5}^{+2.6} \pm 2.5\right) \times 10^{-6}$. The longitudinal polarization fractions are measured to be $\Gamma_{L} / \Gamma\left(\phi K^{* 0}\right)=$ $0.65 \pm 0.07 \pm 0.04$ and $\Gamma_{L} / \Gamma\left(\phi K^{*+}\right)=0.46 \pm 0.12 \pm 0.05$. We measure the charge asymmetries: $\mathcal{A}_{C P}\left(\phi K^{* 0}\right)=+0.04 \pm 0.12 \pm 0.02$ and $\mathcal{A}_{C P}\left(\phi K^{*+}\right)=+0.16 \pm 0.17 \pm 0.04$.
\end{abstract}

Presented at the XVII ${ }^{\text {th }}$ Rencontres de la Vallée d'Aoste, $3 / 9-3 / 15 / 2003$, La Thuile, Vallée d'Aoste, Italy

Stanford Linear Accelerator Center, Stanford University, Stanford, CA 94309

Work supported in part by Department of Energy contract DE-AC03-76SF00515.

Work supported by US DOE contract W-7405-ENG-48, Univ. of CA, Lawrence Livermore National Laboratory. 


\section{INTRODUCTION}

The first evidence for charmless vector-vector $B$ decays was provided by the CLEO experiment with the measurement of one $B \rightarrow \phi K^{*}$ channel [1]. Measurement of both $B \rightarrow \phi K^{*}$ charge modes was performed by $B A B A R$ [2], which also reported the search for direct $C P$ violation in these modes [3]. The CLEO experiment also set upper limits on the decay rates in several other vector-vector final states of $B$ decays [4]. The Belle experiment announced large signal yield in the $B^{+} \rightarrow \rho^{0} \rho^{+}$ channel [5].

Recently, there has been interest in charmless $B$ decays because of the clean environment for the search for new physics. For example, new particles contributing to penguin diagrams, such as charged Higgs bosons or SUSY particles, would provide additional amplitudes with different phases. Charmless $B$ decays are also sensitive to the weak phases $\alpha \equiv \arg \left[-V_{t d} V_{t b}^{*} / V_{u d} V_{u b}^{*}\right]$ and $\gamma \equiv \arg \left[-V_{u d} V_{u b}^{*} / V_{c d} V_{c b}^{*}\right]$ arising from the elements of the Cabibbo-Kobayashi-Maskawa (CKM) mixing matrix [6]. The vector-vector charmless $B$ decays provide additional information about the decay dynamics and strong phases, which could be obtained from the analysis of angular distributions [7].

The decays $B \rightarrow \phi K^{*}$ are expected to proceed through pure penguin diagrams ( $b \rightarrow s$ loops) as illustrated in Fig. 1. Similarly, the decays $B \rightarrow \rho K^{*}$ are expected to be dominated by $b \rightarrow s$ penguin transitions with additional contributions from Cabibbo-suppressed tree-level $b \rightarrow u$ transitions, while the decays $B \rightarrow \rho \rho$ proceed primarily through Cabibbo-favored tree-level $b \rightarrow u$ transitions and CKM-suppressed $b \rightarrow d$ penguins.

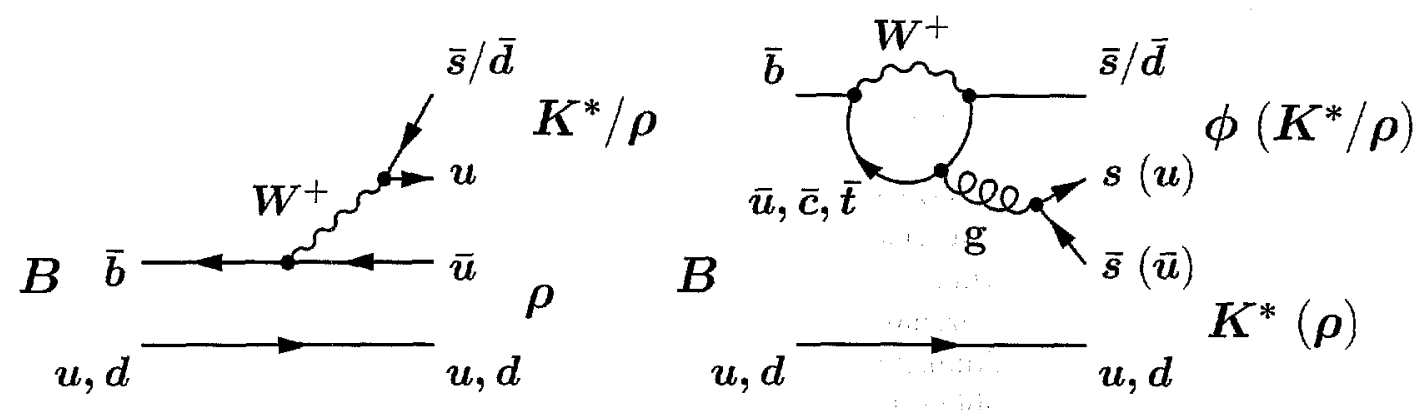

Figure 1: Two of the dominant diagrams describing the decays $B \rightarrow \rho \rho, \rho K^{*}$, and $\phi K^{*}$.

The measurement of direct $C P$ violation in pure penguin modes, such as $B \rightarrow \phi K^{*}$, is sensitive to non-standard-model physics [8]. In the standard model, direct $C P$ violation could arise due to the difference between the $b \rightarrow u$ tree and $b \rightarrow s(b \rightarrow d)$ penguin amplitude weak phases [9], which is $\gamma(\alpha)$ in the case of the decays $B \rightarrow \rho K^{*}(B \rightarrow \rho \rho)$. Direct $C P$ violation would be observed as an asymmetry of $B$ decay rates:

$$
\mathcal{A}_{C P} \equiv \frac{\Gamma(\bar{B} \rightarrow \bar{f})-\Gamma(B \rightarrow f)}{\Gamma(\bar{B} \rightarrow \bar{f})+\Gamma(B \rightarrow f)} .
$$

However, large uncertainties in the strong phases, which can be calculated by certain models, weakens the quantitative relationship to the weak phases. 
The time-dependent asymmetries in $B$ decays to $C P$ eigenstates would provide important tests of the standard model [10]. Comparison of the value of $\sin 2 \beta$ obtained from $\phi K^{* 0}\left(\rightarrow K_{S}^{0} \pi^{0}\right)$ with that from charmonium modes can probe for new physics. Time-dependent measurements in $B \rightarrow \rho \rho$ modes combined with isospin relations among the decay amplitudes for these modes would provide a measurement of $\alpha$. Angular analysis is important for time-dependent asymmetries because of the mixture of $C P$-odd and $C P$-even components, and for the isospin analysis of $B \rightarrow \rho \rho$ modes.

\section{THE BABAR DETECTOR AND DATASET}

In this analysis we use the data collected with the BABAR detector [11] at the PEP-II asymmetricenergy $e^{+} e^{-}$collider [12] located at the Stanford Linear Accelerator Center. The results presented in this paper are based on data taken in the 1999-2002 run comprising an integrated luminosity of $81.9 \mathrm{fb}^{-1}$, corresponding to 88.9 million $B \bar{B}$ pairs, at the $\Upsilon(4 S)$ resonance (on-resonance) and $9.6 \mathrm{fb}^{-1}$ approximately $40 \mathrm{MeV}$ below this energy (off-resonance). The $\Upsilon(4 S)$ resonance occurs at the $e^{+} e^{-}$center-of-mass (c.m.) energy, $\sqrt{s}$, of $10.58 \mathrm{GeV}$. The asymmetric beam configuration in the laboratory frame provides a boost to the $\Upsilon(4 S)$ increasing the momentum range of the $B$ meson decay products up to about $4 \mathrm{GeV} / c$.

Charged-particle momenta are measured in a tracking system that is a combination of a silicon vertex tracker (SVT) consisting of five double-sided detectors and a 40-layer central drift chamber $(\mathrm{DCH})$, both operating in a 1.5-T solenoidal magnetic field. BABAR achieves an impact parameter resolution of about $40 \mu \mathrm{m}$ for the high momentum charged particles in the $B$ decay final states, allowing the precise determination of decay vertices. The tracking system covers $92 \%$ of the solid angle in the c.m. frame. The average track-finding efficiency is approximately $98 \%$ for momenta above $0.2 \mathrm{GeV} / c$ when the angle between track and the beam axis is greater than $500 \mathrm{mrad}$.

Charged particle identification is provided by the average energy loss $(d E / d x)$ in the tracking devices (SVT and DCH) and by an internally reflecting ring imaging Cherenkov detector (DIRC) covering the central region. A $K-\pi$ separation of better than four standard deviations $(\sigma)$ is achieved for momenta below $3 \mathrm{GeV} / c$, decreasing to $2.5 \sigma$ at the highest momenta in the $B$ decay final states. Photons are detected by a CsI(Tl) electromagnetic calorimeter (EMC), which provides excellent angular and energy resolution with high efficiency for energies above $30 \mathrm{MeV}$. For a $1 \mathrm{GeV}$ photon the energy and angular resolutions are $3 \%$ and $4 \mathrm{mrad}$, respectively. Electrons are identified by the tracking system and the EMC.

\section{EVENT SELECTION}

Hadronic events are selected based on track multiplicity and event topology. We fully reconstruct $B$ meson candidates from their charged and neutral decay products including the intermediate states $\phi \rightarrow K^{+} K^{-}, K^{* 0} \rightarrow K^{+} \pi^{-}, K^{* 0} \rightarrow K^{0} \pi^{0}, K^{*+} \rightarrow K^{+} \pi^{0}, K^{*+} \rightarrow K^{0} \pi^{+}, \rho^{0} \rightarrow \pi^{+} \pi^{-}$, $\rho^{+} \rightarrow \pi^{+} \pi^{0}$, with $\pi^{0} \rightarrow \gamma \gamma$ and $K^{0} \rightarrow K_{S}^{0} \rightarrow \pi^{+} \pi^{-}$[13]. Candidate charged tracks are required to originate from the interaction point, and to have at least $12 \mathrm{DCH}$ hits and a minimum transverse momentum of $0.1 \mathrm{GeV} / c$. Looser criteria are applied to tracks forming $K_{S}^{0}$ candidates to allow for displaced decay vertices. The $K_{S}^{0}$ candidates are required to satisfy $\left|m_{\pi^{+} \pi^{-}}-m_{K^{0}}\right|<12 \mathrm{MeV} / c^{2}$ with the cosine of the angle between their reconstructed flight and momentum directions greater than 0.995 and the measured proper decay time greater than five times its uncertainty. Kaon tracks are distinguished from pion and proton tracks via a likelihood ratio that includes, for momenta 
below $0.7 \mathrm{GeV} / c, d E / d x$ information from the SVT and DCH, and, for higher momenta, the Cherenkov angle and number of photons as measured by the DIRC.

We reconstruct $\pi^{0}$ mesons from pairs of photons, each with a minimum energy $30 \mathrm{MeV}$. The typical width of the reconstructed $\pi^{0}$ mass is $7 \mathrm{MeV} / c^{2} . \mathrm{A} \pm 15 \mathrm{MeV} / c^{2}$ interval is applied to select $\pi^{0}$ candidates. We select $\phi, K^{*}$, and $\rho$ candidates with the following requirements on the invariant masses of their final states: $0.99<m_{K^{+} K^{-}}<1.05,0.75<m_{K \pi}<1.05$, and $0.52<m_{\pi \pi}<1.00$ (all in $\left.\mathrm{GeV} / c^{2}\right)$. The helicity angle $\theta_{\mathrm{x}}$ of a $\phi, K^{*}$, or $\rho(\mathrm{x}=1,2$ for the two resonances in the $B$ decay) is defined as the angle between the direction of one of the two daughters $\left(K^{+}, K\right.$, or $\pi^{+}$respectively) and the parent $B$ direction in the resonance rest frame. To suppress combinatorial background we restrict the $K^{*} \rightarrow K \pi^{0}$ and $\rho^{+} \rightarrow \pi^{+} \pi^{0}$ helicity angles $\left(\cos \theta_{\mathrm{x}}>-0.5\right)$. This effectively requires the $\pi^{0}$ momentum to be larger than $0.35 \mathrm{GeV} / c$.

We identify $B$ meson candidates kinematically using two nearly independent variables [11], the beam energy-substituted mass $m_{\mathrm{ES}}=\left[\left(s / 2+\mathbf{p}_{i} \cdot \mathbf{p}_{B}\right)^{2} / E_{i}^{2}-\mathbf{p}_{B}^{2}\right]^{1 / 2}$ and the energy difference $\Delta E=\left(E_{i} E_{B}-\mathbf{p}_{i} \cdot \mathbf{p}_{B}-s / 2\right) / \sqrt{s}$, where $\left(E_{i}, \mathbf{p}_{i}\right)$ is the initial state four-momentum obtained from the beam momenta, and $\left(E_{B}, \mathbf{p}_{B}\right)$ is the four-momentum of the reconstructed $B$ candidate. For signal events $m_{\mathrm{ES}}$ peaks at the $B$ mass and $\Delta E$ at zero. Our initial selection requires $m_{\mathrm{ES}}>5.2$ $\mathrm{GeV} / \mathrm{c}^{2}$ and $|\Delta E|<0.2 \mathrm{GeV}$.

Charmless hadronic modes suffer from a large background due to random combinations of tracks produced in quark-antiquark continuum events $\left(e^{+} e^{-} \rightarrow q \bar{q}, q=u, d, s, c\right)$. Background events from the continuum are distinguished by a jet-like structure as opposed to the more spherical topology of $B \bar{B}$ pairs produced in $\Upsilon(4 S)$ events. To reject continuum background we require $\left|\cos \theta_{T}\right|<0.8$, where $\theta_{T}$ is the angle between the thrust axis of the $B$ candidate and that of the rest of the tracks and neutral clusters in the event, calculated in the c.m. frame. The distribution of the $\cos \theta_{T}$ variable is sharply peaked near \pm 1 for combinations drawn from jet-like $q \bar{q}$ pairs, and nearly uniform for the isotropic $B$ meson decays. We also construct a Fisher discriminant that combines eleven variables [14]: the polar angles of the $B$ momentum vector and the $B$-candidate thrust axis with respect to the beam axis in the $\Upsilon(4 S)$ frame, and the scalar sum of the c.m. momenta of charged particles and photons (excluding particles from the $B$ candidate) entering nine coaxial angular intervals of $10^{\circ}$ around the $B$-candidate thrust axis.

Monte Carlo (MC) simulation [15] demonstrates that contamination from other $B$ decays is negligible for the modes with narrow $\phi$ resonance and is relatively small for other charmless $B$ decay modes. We achieve further suppression of $B$ decay background by removing all signal candidates that have decay products consistent with $D \rightarrow K \pi, K \pi \pi$ decays. The remaining small background coming from $B$ decays is accounted for in the fit. It is found that background subtraction is necessary only in the $B \rightarrow \rho K^{*}$ analysis, where there is a small probability of charmed $B$ decays being reconstructed as signal. In this analysis we assume negligible contribution of other partial waves in our final states selected within vector resonance mass windows.

\section{ANALYSIS METHOD}

We use an unbinned extended maximum likelihood (ML) fit to extract signal yields, charge asymmetries, and angular polarizations simultaneously. We define the likelihood for each event candidate:

$$
\mathcal{L}_{i}=\sum_{j=1}^{3} \sum_{k=1}^{2} n_{j k} \mathcal{P}_{j k}\left(\vec{x}_{i} ; \vec{\alpha}\right)
$$


where $\mathcal{P}_{j k}\left(\vec{x}_{i} ; \vec{\alpha}\right)$ is the probability density function (p.d.f.) for measured variables $\vec{x}_{i}$ of a candidate $i$ in category $j$ and flavor state $k$, and $n_{j k}$ are the yields to be extracted from the fit. There are three categories: signal $(j=1)$, continuum $q \bar{q}(j=2)$, and $B \bar{B}$ combinatorial background $(j=3)$. The p.d.f.'s are non-zero only for the correct final state flavor $(k=1$ for $\bar{B} \rightarrow \bar{f}$ and $k=2$ for $B \rightarrow f$ ). The $B$ flavor is determined by its charge, except for the $\phi K^{* 0}$ final state where the flavor is determined from the charge of the kaon from the $K^{* 0} \rightarrow K^{+} \pi^{-}$decay, and the flavor is not defined for the $K^{* 0} \rightarrow K^{0} \pi^{0}$ decay. The fixed parameters $\vec{\alpha}$ define the expected distributions of measured variables in each category and flavor state. We rewrite the event yields $n_{j k}$ in each category in terms of the asymmetry $\mathcal{A}_{j}$ and the total event yield $n_{j}: n_{j 1}=n_{j} \times\left(1+\mathcal{A}_{j}\right) / 2$ and $n_{j 2}=n_{j} \times\left(1-\mathcal{A}_{j}\right) / 2$. This definition is consistent with Eq. 1 .

The fit input variables $\vec{x}_{i}$ are $\Delta E, m_{\mathrm{ES}}$, Fisher discriminant, invariant masses of the $K^{*}$ and $\phi$ (or $\rho$ ) resonances, and the $K^{*}$ and $\phi$ (or $\rho$ ) helicity angles $\theta_{\mathrm{x}}(\mathrm{x}=1,2)$. The correlations among the fit input variables in the data and signal MC are found to be small (typically less than $5 \%$ ). The p.d.f. $\mathcal{P}_{j k}\left(\vec{x}_{i} ; \vec{\alpha}\right)$ for a given candidate $i$ is the product of the p.d.f.'s for each of the variables, except for the helicity angles. We take into account the angular correlations in the signal and the detector acceptance effects in the helicity angle p.d.f. parameterization. Due to the limited statistics in our analysis we adopt a simplified angular analysis technique where we integrate over the angle between the decay planes of the two vector-particle decays, leaving a p.d.f. that depends only on the two helicity angles. This distribution is sufficient to determine the longitudinal polarization fraction $f_{L} \equiv \Gamma_{L} / \Gamma$. The differential decay width is defined as:

$$
\frac{1}{\Gamma} \frac{d^{2} \Gamma}{d \cos \theta_{1} d \cos \theta_{2}}=\frac{9}{4}\left\{\frac{1}{4}\left(1-f_{L}\right) \sin ^{2} \theta_{1} \sin ^{2} \theta_{2}+f_{L} \cos ^{2} \theta_{1} \cos ^{2} \theta_{2}\right\} .
$$

We allow for multiple candidates in a given event by assigning candidates a weight of $1 / N_{i}$, where $N_{i}$ is the number of candidates in the same event. The average number of candidates per event is close to one (varying from 1.05 to 1.14 depending on the mode). The extended likelihood for a sample of $N_{\text {cand }}$ candidates is

$$
\mathcal{L}=\exp \left(-\sum_{j=1}^{3} n_{j}\right) \prod_{i=1}^{N_{\text {cand }}} \exp \left(\frac{\ln \mathcal{L}_{i}}{N_{i}}\right) .
$$

The event yields $n_{j}$, asymmetries $\mathcal{A}_{j}$, and polarization $f_{L}$ are obtained by minimizing the quantity $\chi^{2} \equiv-2 \ln \mathcal{L}$ using the minimization package MINUIT [16]. The dependence of $\chi^{2}$ on a fit parameter $n_{j}, \mathcal{A}_{j}$, or $f_{L}$ is obtained with the other fit parameters floating. We quote statistical errors corresponding to unit change in $\chi^{2}$. When more than one $K^{*}$ decay channel is measured for the same $B$ decay, the channels are combined by adding their $\chi^{2}$ distributions for $n_{j}, \mathcal{A}_{j}$, or $f_{L}$. The statistical significance of a signal is defined as the square root of the change in $\chi^{2}$ when constraining the number of signal events to zero in the likelihood fit; it describes the probability for the background to fluctuate to the observed event yield.

The fixed parameters $\vec{\alpha}$ describing the p.d.f.'s for signal and background distributions are extracted from MC simulation, on-resonance $\Delta E-m_{\mathrm{ES}}$ sidebands, and off-resonance data. The MC resolutions are adjusted by comparisons of data and simulation in abundant calibration channels with similar kinematics and topology, such as $B \rightarrow D \pi, D \rho$ with $D \rightarrow K \pi \pi, K \pi$. The simulation reproduces the event-shape variable distributions found in data.

To describe the signal distributions, we employ Gaussian functions for the parameterization of the p.d.f.'s for $\Delta E$ and $m_{E S}$ and a relativistic $P$-wave Breit-Wigner distribution convoluted 
with a Gaussian resolution function for the resonance masses. For the background we use lowdegree polynomials or, in the case of $m_{\mathrm{ES}}$, an empirical phase-space function [17]. The background parameterizations for resonance masses also include a resonant component to account for resonance production in the continuum. The background helicity angle distribution shape is again separated into contributions from combinatorics and from real mesons, both fit by low-degree polynomials multiplied by an empirical function $1 /\left(1+\exp \left(\left(\theta_{\mathrm{x}}-\theta_{0}\right) / a\right)\right)$ to account for the detector acceptance effects, where $\theta_{0}$ and $a$ are fixed parameters. For both the signal and background, the p.d.f. for the Fisher discriminant is represented by a Gaussian with different widths above and below the mean.

\section{PHYSICS RESULTS AND SYSTEMATIC UNCERTAINTIES}

The results of our maximum likelihood fits are summarized in Table 1. For the branching fractions we assume equal production rates of $B^{0} \bar{B}^{0}$ and $B^{+} B^{-}$. We find significant signals in both $\phi K^{*}$ decay modes. We measure the charge asymmetries and longitudinal polarizations in all $\phi K^{*}$ final states. We also observe a significant yield of events in $\rho^{0} K^{*+}(4.7 \sigma)$ and $\rho^{0} \rho^{+}(4.4 \sigma)$ final states. The projections of the fit results are shown in Fig. 2 and 3, where we plot only a subsample of events, enhancing the signal with a requirement on the ratio of the signal probability to background probability $\left(\mathcal{P}_{\text {sig }}\right.$ and $\mathcal{P}_{\text {bkg }}$ from Eq. 2).

We study the performance of the ML fit with the MC samples where the signal events are taken from the complete MC simulation and the background is distributed according to the sideband parameterizations. The input values for the number of signal events, charge asymmetry, and

Table 1: Summary of results for the measured $B$ decay modes; $\varepsilon$ denotes the reconstruction efficiency and $\varepsilon_{\text {tot }}$ the total efficiency including daughter branching fractions, both in percent; $n_{\text {sig }}$ is the fitted number of signal events, $\mathcal{B}$ is the branching fraction, $\mathcal{A}_{C P}$ is the signal charge asymmetry, and $f_{L}$ is the longitudinal polarization. The decay channels of $K^{*}$ are shown when more than one final state is measured for the same $B$ decay mode. All results include systematic errors, which are quoted following the statistical errors.

\begin{tabular}{ccccccc}
\hline \hline Mode & $\varepsilon$ & $\varepsilon_{\text {tot }}$ & $n_{\text {sig }}$ & $\mathcal{B}\left(\times 10^{-6}\right)$ & $\mathcal{A}_{C P}$ & $f_{L}$ \\
\hline$\phi K^{*+}$ & - & 5.3 & - & $12.1_{-1.9}^{+2.1} \pm 1.5$ & $+0.16 \pm 0.17 \pm 0.04$ & $0.46 \pm 0.12 \pm 0.05$ \\
\hline$\rightarrow K^{0} \pi^{+}$ & 26.0 & 2.9 & $33.3_{-6.4}^{+7.2} \pm 1.2$ & $12.7_{-2.5}^{+2.8} \pm 1.2$ & $-0.02 \pm 0.20 \pm 0.03$ & $0.50_{-0.15}^{+0.14} \pm 0.04$ \\
$\rightarrow K^{+} \pi^{0}$ & 14.3 & 2.3 & $22.3_{-6.5}^{+7.5} \pm 3.2$ & $10.7_{-3.1}^{+3.6} \pm 1.9$ & $+0.63_{-0.31}^{+0.25} \pm 0.05$ & $0.40_{-0.19}^{+0.20} \pm 0.07$ \\
\hline$\phi K^{* 0}$ & - & 10.2 & - & $11.1_{-1.2}^{+1.3} \pm 1.1$ & $+0.04 \pm 0.12 \pm 0.02$ & $0.65 \pm 0.07 \pm 0.04$ \\
\hline$\rightarrow K^{+} \pi^{-}$ & 29.7 & 9.7 & $101_{-11}^{+12} \pm 3$ & $11.7 \pm 1.4 \pm 1.1$ & $+0.04 \pm 0.12 \pm 0.02$ & $0.64 \pm 0.07 \pm 0.03$ \\
$\rightarrow K^{0} \pi^{0}$ & 11.4 & 0.6 & $2.0_{-1.3}^{+3.4} \pm 0.6$ & $3.5_{-2.3}^{+6.1} \pm 1.1$ & - & $1.00_{-0.66}^{+0.00} \pm 0.25$ \\
\hline$\rho^{0} K^{*+}$ & - & 8.4 & - & $7.7_{-2.0}^{+2.1} \pm 1.4$ & - & - \\
\hline$\rightarrow K^{0} \pi^{+}$ & 21.0 & 4.8 & $44.4_{-11.4}^{+12.5} \pm 3.4$ & $10.4_{-2.7}^{+2.9} \pm 1.7$ & - & - \\
$\rightarrow K^{+} \pi^{0}$ & 10.9 & 3.6 & $9.1_{-9.6}^{+11.1} \pm 5.2$ & $2.9_{-3.0}^{+3.5} \pm 1.8$ & - & - \\
\hline$\rho^{0} \rho^{+}$ & 11.3 & 11.1 & $97.5_{-24.3}^{+26.1} \pm 12.1$ & $9.9_{-2.5}^{+2.6} \pm 2.5$ & - & - \\
\hline \hline
\end{tabular}



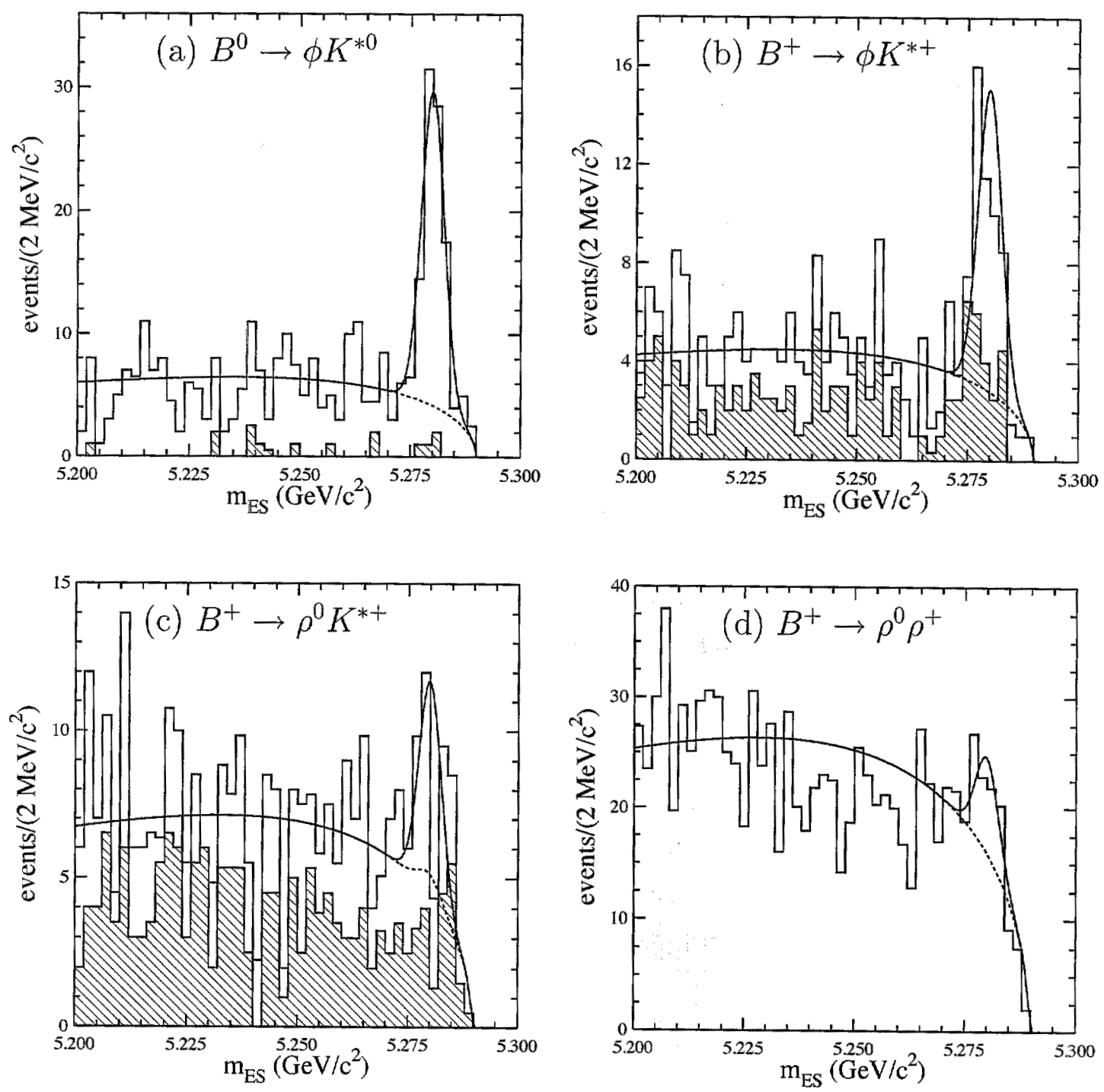

Figure 2: Projections onto the variable $m_{\mathrm{ES}}$ for $B^{0} \rightarrow \phi K^{* 0}$ (a), $B^{+} \rightarrow \phi K^{*+}$ (b), $B^{+} \rightarrow \rho^{0} K^{*+}$ $(c)$, and $B^{+} \rightarrow \rho^{0} \rho^{+}(d)$ candidates after a requirement on the signal-to-background probability ratio $\mathcal{P}_{\text {sig }} / \mathcal{P}_{\mathrm{bkg}}$ with the p.d.f. for $m_{\mathrm{ES}}$ excluded. The histograms show the data, which are the sum of the two $K^{*}$ decay channels when appropriate, while the shaded area is $K^{*} \rightarrow K \pi^{0}$ channel alone. The solid (dashed) line shows the signal-plus-background (background only) p.d.f. projection. 

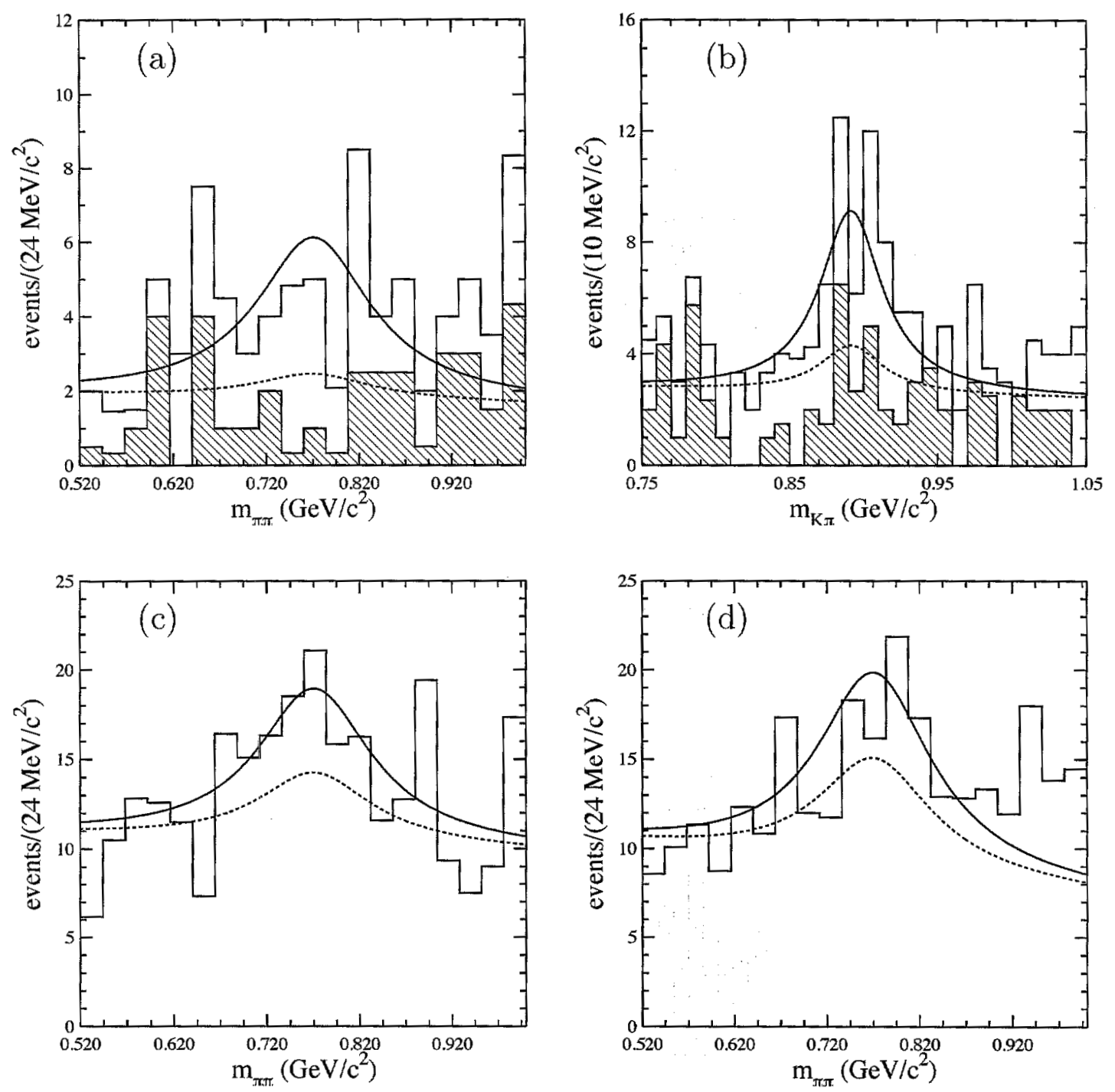

Figure 3: Vector resonance invariant mass distributions for $B^{+} \rightarrow \rho^{0} K^{*+}$ candidates: $m_{\pi^{+} \pi^{-}}$(a) and $m_{K \pi}(b)$; and for $B^{+} \rightarrow \rho^{0} \rho^{+}$candidates: $m_{\pi^{+} \pi^{-}}$(c) and $m_{\pi^{+} \pi^{0}}(d)$, after a requirement on the signal-to-background probability ratio $\mathcal{P}_{\mathrm{sig}} / \mathcal{P}_{\mathrm{bkg}}$ with the p.d.f. for the invariant mass excluded. The histograms show the data and the shaded area corresponds to the $\rho^{0} K^{*+}$ final state with $K^{*+} \rightarrow K^{+} \pi^{0}$ channel alone. The solid (dashed) line shows the signal-phus-background (background only) p.d.f. projection. 
decay polarization are well reproduced, and any small discrepancy is accounted for in the systematic errors.

Systematic uncertainties in the ML fit originate from assumptions about the signal and background distributions. Uncertainties in the p.d.f. parameters arise from the limited statistics in the background sideband data and signal control samples. We vary the p.d.f. parameters within their respective uncertainties, and derive the associated systematic errors. The signals remain statistically significant under these variations. Additional systematic errors in the number of signal events originate from uncertainty in the charm background subtraction in the $\rho K^{*}$ channels where we take the uncertainty to be $100 \%$ of the subtracted values ( 2 and 5 events in $K^{*+} \rightarrow K^{0} \pi^{+}$and $K^{*+} \rightarrow K^{+} \pi^{0}$ channels respectively).

The dominant systematic errors in the efficiency are track finding $(0.8 \%$ per track), particle identification (2\% per track), and $K_{S}^{0}$ and $\pi^{0}$ reconstruction (5\% each). Other minor systematic effects from event selection criteria, daughter branching fractions [18], $\mathrm{MC}$ statistics, and $B$ meson counting sum to less than $4 \%$. The efficiency in the ML fit to signal samples can be less than $100 \%$ because of fake combinations passing the selection criteria, and we account for this with a systematic uncertainty (3-12\%). This effect is larger in the final states with $\rho$ resonances because of the broader distributions. Efficiency uncertainties affect the value of the branching fraction, but not its significance.

In the search for $\rho K^{*}$ and $\rho \rho$ final states we fit only for event yields and exclude angular and $B$-flavor information from the analysis. The reconstruction efficiency depends on the decay polarization. We average reconstruction efficiencies for the $100 \%$ transverse and $100 \%$ longitudinal angular polarizations for each decay channel and assign the systematic errors as the root-mean-square of the uniform efficiency distributions between the two extreme cases. The resulting systematic errors are $9 \%$ in $\rho^{0} K^{*+}\left(\rightarrow K^{0} \pi^{+}\right), 19 \%$ in $\rho^{0} K^{*+}\left(\rightarrow K^{+} \pi^{0}\right)$, and $18 \%$ in $\rho^{0} \rho^{+}$decay channels. For the $\phi K^{*}$ final states we calculate the efficiencies assuming an average polarization of $(0.60 \pm 0.06 \pm 0.05)$ and assign a systematic error corresponding to the total polarization measurement error.

In the polarization measurements, we again include uncertainties from p.d.f. variations where we account for possible bias in the detector acceptance and background parameterizations. The biases from the finite resolution in helicity angle measurement and dilution due to the presence of the fake combinations are studied with MC simulation and are accounted for with conservative systematic error of 0.03 .

We find the charge asymmetry of the track reconstruction efficiency to be consistent with zero within an uncertainty of less than 0.01 for a wide range of momenta [3]. Taking into account particle identification requirements similar to the ones applied to the $K^{*}$ daughters, the asymmetry is consistent with zero with an uncertainty of 0.02 . We also find a negligible effect on the measured asymmetry from any possible bias in track-momentum measurements studied in $e^{+} e^{-} \rightarrow \mu^{+} \mu^{-}$and cosmic ray events. The asymmetry measurement in the $B^{0} \rightarrow \phi K^{* 0}$ decay mode is corrected by the inverse dilution factor $1 /(1-2 w)$, where $w$, the fraction of doubly misidentified $K \pi$ combinations originating from $K^{* 0}$, is less than 0.01 .

\section{SUMMARY}

We have measured branching fractions, longitudinal polarizations, and charge asymmetries with the decays $B^{0} \rightarrow \phi K^{* 0}$ and $B^{+} \rightarrow \phi K^{*+}$. Because the final states contain three strange quarks or antiquarks, in the standard model they are necessarily due to penguin diagrams. This makes them particularly susceptible to non-standard-model contributions. We observe the decays $B^{+} \rightarrow \rho^{0} K^{*+}$ 
and $B^{+} \rightarrow \rho^{0} \rho^{+}$and report the corresponding branching fractions. The $B^{+} \rightarrow \rho^{0} K^{*+}$ decay process is of interest for direct $C P$ measurements due to possible large penguin-tree interference, while the $B \rightarrow \rho \rho$ decays have potential for the measurement of the weak phase $\alpha$.

The $B \rightarrow \phi K^{*}$ branching fractions are in agreement with our earlier less precise measurements [2]. Our charge asymmetry results rule out a significant part of the physical $\mathcal{A}_{C P}$ region, allowing for constraints on new physics models [8], but are not yet of sufficient precision to allow precise comparison with standard model predictions [19]. We have performed the angular analysis in the penguin-dominated rare $B$ decays and measure a relatively large longitudinal polarization in the decay amplitude, as predicted [7]. Our measurement of $B^{+} \rightarrow \rho^{0} \rho^{+}$branching fraction is significantly lower than the central value measured by Belle [5] even taking into account polarization uncertainty. Our results are preliminary and use increased statistics compared to the earlier $B A B A R$ measurements of the $B \rightarrow \phi K^{*}$ branching fractions and charge asymmetries $[2,3]$.

\section{ACKNOWLEDGMENTS}

We are grateful for the extraordinary contributions of our PEP-II colleagues in achieving the excellent luminosity and machine conditions that have made this work possible. The success of this project also relies critically on the expertise and dedication of the computing organizations that support BABAR. The collaborating institutions wish to thank SLAC for its support and the kind hospitality extended to them. This work is supported by the US Department of Energy and National Science Foundation, the Natural Sciences and Engineering Research Council (Canada), Institute of High Energy Physics (China), the Commissariat à l'Energie Atomique and Institut National de Physique Nucléaire et de Physique des Particules (France), the Bundesministerium für Bildung und Forschung and Deutsche Forschungsgemeinschaft (Germany), the Istituto Nazionale di Fisica Nucleare (Italy), the Foundation for Fundamental Research on Matter (The Netherlands), the Research Council of Norway, the Ministry of Science and Technology of the Russian Federation, and the Particle Physics and Astronomy Research Council (United Kingdom). Individuals have received support from the A. P. Sloan Foundation, the Research Corporation, and the Alexander von Humboldt Foundation.

\section{References}

[1] The CLEO Collaboration, S. Chen et al., Phys. Rev. Lett. 85. 525 (2000).

[2] The BABAR Collaboration, B. Aubert et al., Phys. Rev. Lett. 87, 151801 (2001).

[3] The BABAR Collaboration, B. Aubert et al., Phys. Rev. D 65, 051101 (2002).

[4] The CLEO Collaboration, R. Godang et al., Phys. Rev. Lett. 88, 021802 (2002).

[5] The Belle Collaboration, K. Abe et al., BELLE-CONF-0255.

[6] M. Kobayashi and T. Maskawa, Prog. Theor. Phys. 49, 652 (1973).

[7] G. Kramer, W.F. Palmer, Phys. Rev. D45, 193 (1992); R. Aleksan et al., Phys. Lett. B 356, 95 (1995); C.-H. Chen, Y.-Y. Keum, H.-n. Li, Phys. Rev. D 66, 054013 (2002).

[8] I. Hinchliffe, N. Kersting, Phys. Rev. D 63, 015003 (2001). 
[9] M. Bander, D. Silverman, and A. Soni, Phys. Rev. Lett. 43, 242 (1979).

[10] A.B. Carter and A.I. Sanda, Phys. Rev. D23, 1567 (1981); I.I. Bigi and A.I. Sanda, Nucl. Phys. B193, 85 (1981).

[11] The BABAR Collaboration, B. Aubert et al., Nucl. Instrum. Methods A479, 1 (2002).

[12] PEP-II Conceptual Design Report, SLAC-R-418 (1993).

[13] Inclusion of the charge conjugate states is implied.

[14] The CLEO Collaboration, D.M. Asner et al., Phys. Rev. D 53, 1039 (1996).

[15] The BABAR detector Monte Carlo simulation is based on GEANT: R. Brun et al., CERN $\mathrm{DD} / \mathrm{EE} / 84-1$.

[16] F. James, CERN Program Library, D506.

[17] The ARGUS Collaboration, H. Albrecht et al., Phys. Lett. B 241, 278 (1990).

[18] The Particle Data Group, K. Hagiwara et al., Phys. Rev. D 66, 010001 (2002).

[19] G. Kramer, W.F. Palmer, and H. Simma, Nucl. Phys. B 428, 77 (1994); A. Ali, G. Kramer, and C.-D. Lü, Phys. Rev. D 59, 014005 (1998). 